BULL. AUSTRAL. MATH. SOC.

$05 C 65,05 C 99$

VOL. $26(1982), 479-480$.

\title{
DEGREE MULTISETS OF HYPERGRAPHS
}

\section{David Billington}

A multiset is a "set" which may have repeated elements. If $s$ is a positive integer then an s-uniform hypergraph is a hypergraph in which every block, or edge, contains exactly $s$ points. A hypergraph in which every block contains at least $s$ points is called an $\boldsymbol{s}^{+}$-hypergraph. Let $R(\Delta, s)$ denote the set of all s-uniform hypergraphs which have $\Delta$ as their multiset of degrees. Similarly $R\left(\Delta, s^{+}\right)$denotes the set of all $s^{+}$-hypergraphs which have $\Delta$ as their degree multiset. We make $R(\Delta, s)$ into a graph by defining two elements of $R(\Delta, s)$ to be adjacent if and only if one can be obtained from the other by a very simple operation called an exchange. By considering the components of $R(\Delta, s)$ we are able to make $R\left(\Delta, 8^{+}\right)$into a suitable graph.

In this thesis we investigate the structure of the graphs $R(\Delta, 8)$ and $R\left(\Delta, s^{+}\right)$when $\Delta$ is countable. When $\Delta$ is finite we also consider the structure of two subgraphs of $R(\Delta, 2)$.

Necessary and sufficient conditions on $\Delta$ and $s$ are found for both $R(\Delta, s)$ and $R\left(\Delta, s^{+}\right)$to be non-empty. To find these conditions we first construct canonical elements of $R(\Delta, s)$ and $R\left(\Delta, s^{+}\right)$. If $\Delta$ is denumerable then we determine the number of components and the number of isolated vertices of both $R(\Delta, s)$ and $R\left(\Delta, s^{+}\right)$. When $\Delta$ is finite we show that $R(\Delta, \delta)$ is connected. The definition of $R\left(\Delta, \sigma^{+}\right)$makes it connected when $\Delta$ is finite. All the finite multisets, $\Delta$, for which either $R(\Delta, 6)$ or $R\left(\Delta, \delta^{+}\right)$has exactly one element, are given explicitly. To conclude our study of $R(\Delta, s)$ for arbitrary $s$ and

Received 2 September 1982. Thesis submitted to University of Melbourne, December 1981. Degree approved July 1982. Supervisor: Dr Derek A. Holton. 
finite $\Delta$ we present some necessary conditions on $\Delta$ and $s$ for $R(\Delta, s)$ to be a tree.

Finally we turn our attention to $R(\Delta, 2)$ when $\Delta$ is finite. The vertices of $R(\Delta, 2)$ are just the multigraphs which realise $\Delta$. For any positive integer $m$, an $m$-graph is a multigraph which has at most $m$ edges between any two points. By an exact $m$-graph we mean an $m$-graph in which there exist two points which have exactly $m$ edges between them. The subgraph of $R(\Delta, 2)$ induced by the m-graphs is denoted by $R(\Delta, L(m))$, while $R(\Delta, E(m))$ denotes the subgraph of $R(\Delta, 2)$ induced by the exact m-graphs.

The proof we give that $R(\Delta, L(m))$ is connected provides best possible upper and lower bounds for the shortest distance between any two vertices of $R(\Delta, L(m))$. Although $R(\Delta, E(m))$ is in general not connected, very weak sufficient conditions on $\Delta$ and $m$ are found which ensure that $R(\Delta, E(m))$ is connected. 